\title{
Improving Middle Square Method RNG Using Chaotic Map
}

\author{
Hamed Rahimov', Majid Babaie ${ }^{1}$, Hassan Hassanabadi ${ }^{2}$ \\ ${ }^{1}$ Department of Computer Engineering, Shahrood University of Technology, Shahrood, Iran \\ ${ }^{2}$ Physics Department, Shahrood University of Technology, Shahrood, Iran \\ E-mail:hrahimov@shahroodut.ac.ir,hrahimov@gmail.com \\ Received January 23, 2011; revised March 10, 2011; accepted March 12, 2011
}

\begin{abstract}
One of the classic approaches in PRNGs is the middle square method in which with a simple mathematical model generating pseudorandom numbers in high speed and minimum correlation between output numbers. Despite these unique characteristics, the method contains weaknesses that a broader application of this algorithm will face. In this paper is studied middle square method and then a logistic chaotic map is introduced with its specific features and its improved weaknesses via using these characteristics. Finally the NIST tests suite s are presented, in order to detect the specific characteristics expected from truly random sequences.
\end{abstract}

Keywords: Middle Square Method (MSM), Random Number Generator, Logistic Map, NIST Tests Suite

\section{Introduction}

Nowadays, the world is connected together by networks. The main problem in the present networks is the security protocols. This is due to growing interest in the use of digital chaotic systems offering the possibility to support the security of cryptographic algorithms for data encrypttion, like those presented in the networking security protocols $[1,2]$.

The best ways to increase security in modern communication methods is using the cryptography algorithms. Most of these new algorithms use chaotic maps as pseudorandom number generators (PRNGs) [3,4] to obtain a binary stream including symmetric encryption algorithm [5]. PRNGs are very important in several fields like statistical studies, simulations, cryptography and solving specific mathematical problems [6-8]. They may be based on unpredictable physical resources such as physical phenomenon or on mathematical algorithms like "Halton algorithm" [6]. However, truly random numbers (i.e. physical phenomenon) are not gained by a specific algorithm $[8,9]$ as they are used in the encryption systems [9]. So we should make use of any real implementation some simple functions for RNG instead of TRNGs [10,11]. A PRNG should be checked by certain statistical tests which it must pass all statistical tests that are restricted to polynomial time in the size of the seed and frequency of " 0 "s and " 1 "s in the specific sequences [12].
In this paper we have presented a chaotic RNG based on middle square method to prepare a mathematical model for generating random numbers. Our model is then tested by the best known statistical method, i.e. the NIST test suite.

One of the fundamental subjects in generating random numbers is the Uniform distribution [11] in the interval $[0,1]$ (e.g. generating real numbers like: 0.0293 or 0.8934). Generating in this interval enables us to investigate our practical purposes for introducing an efficient encryption method. The reason is that the samples from the other distributions are derived using the Uniform Distribution [11]. So it is important that our proposed generator has the uniform distribution.

This part introduces a brief history of some PRNG algorithms, The "Van der Corput" [6], "Halton" [6] and "Sobol" [6] sequences are three basic mathematical algorithms for PRNGs. These are based on low discrepancy method with which unique properties are used in the effective areas such as army industry. Nevertheless, they have some disadvantages in high dimensions, an example being the generated numbers in $20 \times 21$ dimensions for Halton sequence due to multidimensional clustering [6] (as shown in Figure 1).

In order to clarify the purpose of the proposed algorithm, one can see some of methods in References [13-27].

With this background, it is understood that in the recent years the PRNG's algorithm developed by some 


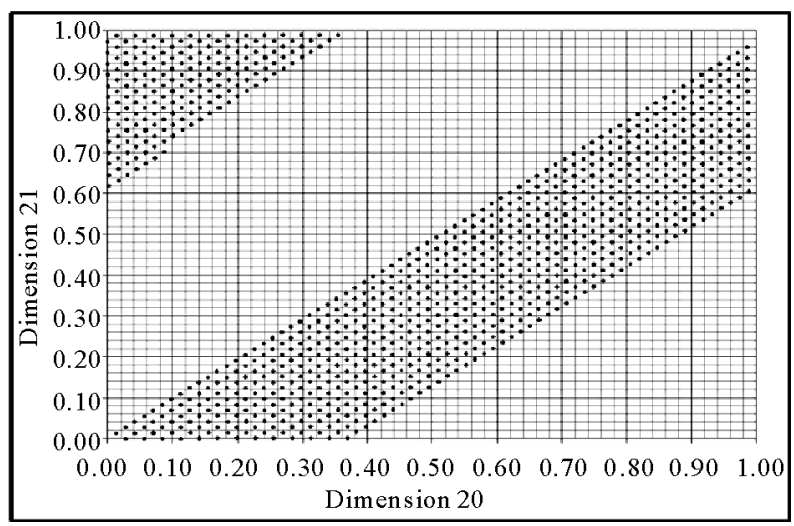

Figure 1. Halton sequences multidimensional clustering: Dimensions $20 \times 21$.

physical algorithm such as chaos theory and produced random numbers rapidly and with less correlation [1327]. This paper investigates an efficient method to produce random numbers with the combination of middle Square Method (MSM) and chaotic logistic map. Hence in the next section, we describe the random number's application in the cryptography algorithm.

\section{Middle Square Method}

Middle Square Method is a one of the best methods of generating pseudorandom numbers. But in the some practical situations it is not a good method, since its period is usually very short (as shown in the Figure 2 and Figure 3). This mathematical algorithm was described by John von Neumann in 1949 [28].

In this method for generating a sequence of ten digit pseudorandom numbers, in the first step a four digits starting value is created and squared (e.g. $A \times A=C$ ) so that an eight digits number is produced. Then by a simple mathematical model generated the middle four digits of the result and it would be the next number in the sequence (e.g. $D=C \bmod 10^{n / 2}$ ) and returned as the result. By repeating this process we are able to generate more random numbers. One of the main problems occurs when the middle $\mathrm{n} / 2$ digits (in this example, four digits) are all zero and the generator yields zero outputs forever. For testing this algorithm we take some random numbers and describe the weaknesses in our examples which would describe $a=8439$ for first example (as shown in Figure 2) and $a=6395$ for second example (as shown in Figure 3) [28].

Sequence one, with core number (8439) according with Figure 2. After generating 214 random numbers we have an unlimited loop generating " 0 ".

Bearing in mind the limited random numbers generated by this method, in this paper proposed a novel

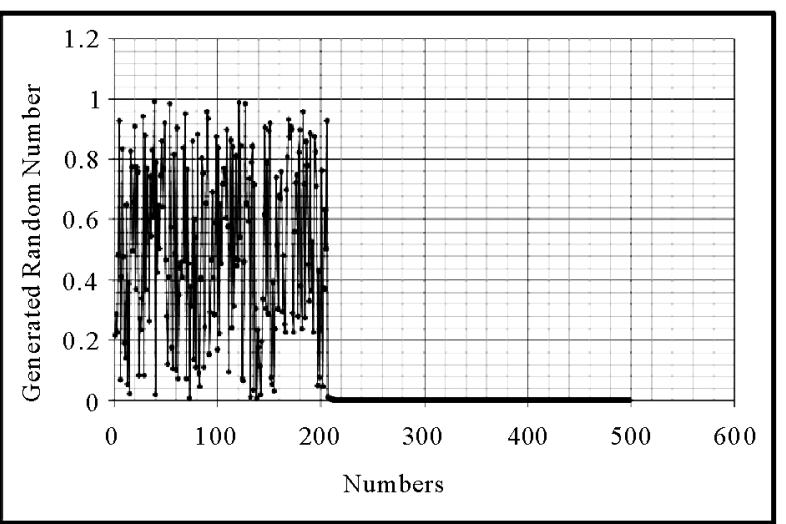

Figure 2. MSM random sequence $($ With core $=8439)$.

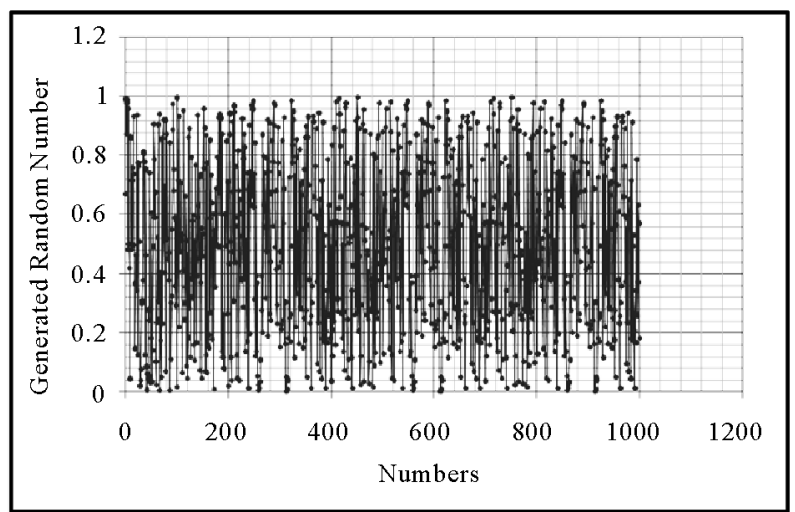

Figure 3. MSM random sequence $($ With core $=6395)$.

chaotic system based on logistic map.

Most of these discrete chaotic cryptosystem algorithms use a chaotic map as PRNGs to generate a keystream which is used for encryption of a plaintext message to produce a ciphertext [15]. The secret key of such systems constitutes the initial values and/or the system parameters [5]. In this paper, a novel sequence generator is proposed which is based on a single one-dimensional logistic map.

Sequence three, with core numbers (6395); according with Figure 3 after generating 216 random numbers we have a loop with length 602 (i.e. where numbers: "217" and " 819 ", we have generated " 0.125 ", so the length of the loop is 602).

This paper is structured as follows. In Section 3, the properties of the logistic map are discussed. In Section 4, a complete description of the chaotic middle square method is presented. The statistical properties of these sequences are discussed in Section 5 and finally the re- sult of NIST test presented in Table $\mathbf{1 .}$

\section{Chaotic Logistic Map}

As we know, the logistic map is the one of the simplest 
and most studied nonlinear that was introduced as a demographic model. The logistic map is given by that is shown in Equation (1):

$$
f\left(x_{n}\right)=x_{n+1}=r \cdot x_{n} \cdot\left(1-x_{n}\right)
$$

Where $\mathrm{xn}$ is the state variable, which generates values in the interval $[0,1]$ and $r$ is called system parameter which can have any value between one and four $[10,29]$.

There is a non-trivial fixed point where $3.4<r \leq 4$ which is seen in Figure 4. Finally for $r=4$, we observe that chaos values are generated in the complete range of $[0,1]$. In this paper, the logistic map has application of

Table 1. Result of NIST tests suite for proposed RNG.

\begin{tabular}{ccc}
\hline Test & P-value & Pass rate \\
\hline Frequency & 0.804645 & 0.9890 \\
Block-Frequency & 0.322901 & 0.9900 \\
CuSums-forward & 0.265567 & 0.9900 \\
CuSums-backward & 0.090388 & 0.9895 \\
Rans & 0.569766 & 0.9915 \\
Long run & 0.066673 & 0.9940 \\
Rank & 0.248649 & 0.9915 \\
FFT & 0.000159 & 0.9995 \\
Overlapping templates & 0.906745 & 0.9905 \\
Universal & 0.971354 & 0.9885 \\
Approximate entropy & 0.558502 & 0.9925 \\
Serial 1 & 0.357000 & 0.9875 \\
Serial 2 & 0.864494 & 0.9905 \\
Liner complexity & 0.671820 & 0.9920 \\
\hline
\end{tabular}

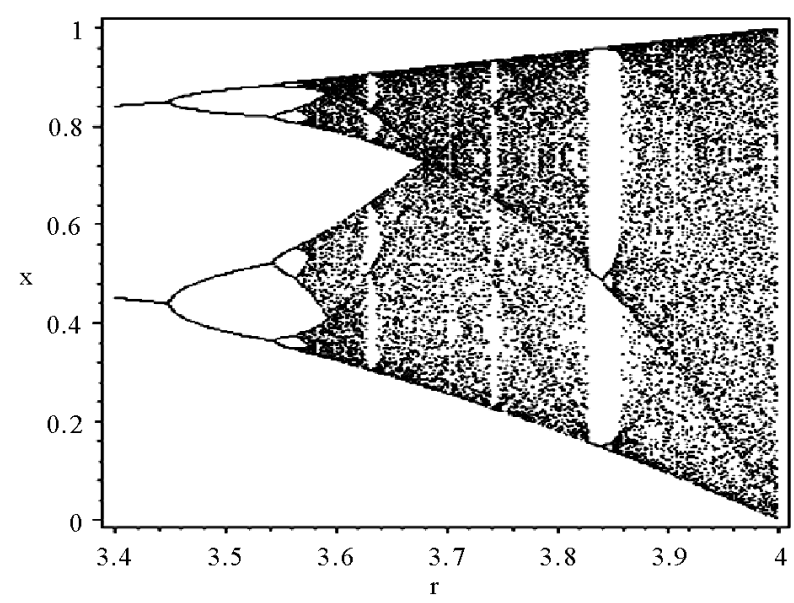

Figure 4. Bifurcation plot of the Logistic map in $x_{n} \in[0,1]$ $r \in[3.4,4]$. the form:

\section{Proposed Chaotic PRNG}

Middle square method has some big problems [28] that were mentioned in Section 3 with the notice of these problems in MSM and consider to unique properties of chaotic logistic map in this section presented a chaotic solution for solving these problems.

When efficient values are chosen for numbers in the core that have no loop with the specific length, next we can correct the unlimited loop (when PRNG joust "0" generated in sequence) by exchanging one of the core's numbers [28].

$$
\begin{aligned}
& C=A \times A \Rightarrow D=C \% 10^{\frac{n}{2}} \\
& E=(C-D) / 10^{\frac{n}{2}} \Rightarrow \text { Result }_{t}=E \% 10^{n} \\
& \text { Num }_{t}={\text { Result } / 10^{n}} \\
& \text { if } \quad\left(\text { Num }_{t}==0\right) \quad \text { then }\{ \\
& \quad x_{n+1}=x_{n} r\left(1-x_{n}\right) \\
& \quad B=x_{n+1} \\
& \quad x_{n}=x_{n+1} \\
& \quad\}
\end{aligned}
$$

Assume the logistic chaotic map with $r=4$ in $x_{0}=0.6$ Generates a random number and replaces it with a number in A's place and continues generating random numbers without any unlimited loop and the loop with a particular length. These problems can be solved by implementation of chaotic iteration that is generated in parallel.

Middle square method algorithm described in Equation (2) also seen below, having the number (i.e. A).

\section{NIST Statistical Tests Suite}

The NIST tests suite is a statistical package involving 15 tests and is based on hypothesis testing. Also The NIST tests suite focus on a variety of different types of non-randomness. In the present case, the tests consist determining whether or not a specific sequence of zeroes and ones is random [8]. The theoretical background of its distribution is based on null hypothesis which is determined by mathematical methods and corresponding probability value (P-value).

The P-value is the probability that a perfect RNG for each test would produce a sequence less random than the sequence that was tested, given the kind of non-randomness assessed by the test. If the P-value for a test is determined to be equal zero, that means the sequence ap- 
pears to be exactly non-random and a P-value to be 1 , then the sequence has perfect randomness [8].

\section{Conclusions}

In this paper we have proposed a pseudorandom number generator (PRNG) based on the combination of chaotic logistic map and Middle Square Method, the chaotic system iterated independently starting from initial conditions could help to generate appropriate values; we have also tested the generated sequences using the NIST tests to detect the unique characteristics expected from truly random bit sequences. The results of statistical testing are reliable so has been used as a part of encryption system to generate secret key and have an efficient algorithm. Finally the result of their statistical tests presented in the Table 1.

\section{References}

[1] Y. Hu, X. Liao, K. W. Wong and Q. Zhou, “A True Random Number Generator Based on Mouse Movement and Chaotic Cryptography," Chaos, Solitons and Fractals, Vol. 40, No. 15, 2009, pp. 2286-2293. doi:10.1016/j.chaos.2007.10.022

[2] P. L. Ecuyer and J. Granger-Piché, "Combined Generators with Components from Different Families," Mathematics and Computers in Simulation, Vol. 62, No. 3, 2003, pp. 395-404. doi:10.1016/S0378-4754(02)00234-3

[3] M. Orlov, “Optimized Random Number Generation in an Interval,” Information Processing Letters, Vol. 109, No. 13, 2009, pp. 722-725. doi:10.1016/j.ipl.2009.03.008

[4] Q. Zhou, X. Liao, K. W. Wong, Y. Hua and D. Xiao, "True Random Number Generator Based on Mouse Movement and Chaotic Hash Function," Information Sciences, Vol. 179, No. 19, 2009, pp. 3442-3450. doi:10.1016/j.ins.2009.06.005

[5] K. Nakano, K. Kawakami and K. Shigemoto, "RSA Encryption and Decryption Using the Redundant Number System on the FPGA," IEEE International Symposium on Parallel \& Distributed Processing, Rome, 23-29 May 2009, pp. 1-8.

[6] I. Krykova, "Evaluating of Path-Dependent Securities with Low Discrepancy Methods," Master of Science Thesis, Worce-Ster Polytechnic Institute, 2003.

[7] T. Stojanovski and L. C. Kocarev, "Chaos-Based Random Number Generators," IEEE Transactions on Circuits and Systems, Vol. 48, No. 3, 2001, pp. 281-288.

[8] K. H. Tsoi, K. H. Leung and P. H. W. Leong, "High Performance Physical Random Number Generator,” IET Proceeding Computers \& Digital Techniques, Vol. 1, No. 4, 2007, pp. 349-352. doi:10.1049/iet-cdt:20050173

[9] M. I. Youssef, M. Zahara, A. E. Emam and M. A. Elghany, "Image Encryption Using Pseudo Random Number and Chaotic Sequence Generators," Radio Science
Conference, New Cairo, 17-19 March 2009, pp. 1-15.

[10] C. Pellicer-Lostao and R. Lopez-Ruiz, "Pseudo-Random Bit Generation Based on 2D Chaotic Maps of Logistic Type and Its Applications in Chaotic Cryptography," Journal of Computational Science and Its Applications, Vol. 5073, 2008, pp. 784-796.

[11] X.-J. Tong, M.-G. Cui and W. Jiang, "The Production Algorithm of Pseudo-Random Number Generator Based on Compound Non-Linear Chaos System," Proceedings of the International Conference on Intelligent Information Hiding and Multimedia Signal Processing, California, 18-20 December 2006, pp. 685-688. doi:10.1109/IIH-MSP.2006.265094

[12] Q. Wang, C. Guyeux and J. M. Bahi, “A Novel PseudoRandom Number Generator Based on Discrete Chaotic Iterations," First International Conference on Evolving Internet, Cannes/La Bocca, 23-29 August 2009, pp. 71-76. doi:10.1109/INTERNET.2009.18

[13] H. P. Lü, S. H. Wang and G. Hu, "Pseudo-Random Number Generator Based on Coupled Map Lattices,” International Journal of Modern Physics B, Vol. 18, No. 17-19, 2004, pp. 2409-2414.

[14] X. M. Li, H. B. Shen and X. L. Yan, "Characteristic Analysis of a Chaotic Random Number Generator Using Piece-Wise-Linear Map,” Journal of Electronics and Information Technology, Vol. 27, No. 6, 2005, pp. 874-878.

[15] J. Liu, "Design of a Chaotic Random Sequence and Its Application,” Computer Engineering, Vol. 31, No. 18, 2005, pp. 150-152.

[16] Y. Wang, H. Shen and X. Yan, "Design of a Chaotic Random Number Generator," Chinese Journal of Semiconductors, Vol. 26, No. 12, 2005, pp. 2433-2439.

[17] L. Wang, F. P. Wang and Z. J. Wang, "Novel ChaosBased Pseudo-Random Number Generator," Acta Physica Sinica, Vol. 55, No. 8, 2006, pp. 3964-3968.

[18] M. Andrecut, "Logistic Map as a Random Number Generator," International Journal of Modern Physics B, Vol. 12, No. 9, 1998, pp. 921-930. doi:10.1142/S021797929800051X

[19] J. A. Gonzalez and R. Pino, "Random Number Generator Based on Unpredictable Chaotic Functions," Computer Physics Communications, Vol. 120, No. 2-3, 1999, pp. 109-114. doi:10.1016/S0010-4655(99)00233-7

[20] L. Kocarev and G. Jakimoski, "Pseudo-Random Bits Generated by Chaotic Maps,” IEEE Transactions on Circuits and Systems, Vol. 50, No. 1, 2003, pp. 123-126. doi:10.1109/TCSI.2002.804550

[21] S. M. Fu, Z. Y. Chen and Y. A. Zhou, "Chaos Based Random Number Generators," Computer Research and Development, Vol. 41, No. 4, 2004, pp. 749-754.

[22] S. Ergun and S. Ozoguz, "Truly Random Number Generators Based on a Non-autonomous Chaotic Oscillator,” AEU-International Journal Electronics \& Communications, Vol. 61, No. 4, 2007, pp. 235-242. doi:10.1016/j.aeue.2006.05.006

[23] V. V. Kolesov, R. V. Belyaev and G. M. Voronov, “A Digital Random-Number Generator Based on the Chaotic 
Signal Algorithm,” Journal of Communications Technology and Electronics, Vol. 46, No. 11, 2001, pp. 12581263.

[24] T. Stojanovski and L. Kocarev, "Chaos-Based Random Number Generators," IEEE Transactions on Circuits and Systems, Vol. 48, No. 3, 2001, pp. 281-288. doi:10.1109/81.915385

[25] T. Stojanovski, J. Pihl and L. Kocarev, "Chaos Based Random Number Generators," IEEE Transactions on Circuits and Systems, Vol. 48, No. 3, 2001, pp. 382-385. doi:10.1109/81.915396

[26] S. Oishi and H. Inoue, "Pseudo-Random Number Generators and Chaos," Transactions of the Institute of Electronics and Communication Engineers of Japan E, Vol. 65, No. 9, 1982, pp. 534-541.
[27] T. Lin and L. O. Chua, "New Class of Pseudo-Random Number Generator Based on Chaos in Digital Filters," International Journal of Circuit Theory and Applications, Vol. 21, No. 5, 1993, pp. 473-480. doi:10.1002/cta.4490210506

[28] W. Gao, G. Y. Zhang, Y. M. Li and W. H. Chen, "Research and Realization of Random Encryption Algorithm," International Conference on Internet Computing in Science and Engineering, Washington, 24 June 2008, pp. 23-26.

[29] J. Szczepa'nski and Z. Kotulski, "Pseudo-Random Number Generators Based on Chaotic Dynamical Systems," Journal of Open Systems \& Information Dynamics, Vol. 8, No. 2, 2001, pp. 137-146. 